\title{
Wedge bronchoplastic lobectomy for non-small cell lung cancer as an alternative to sleeve lobectomy
}

\author{
Seong Yong Park, MD, ${ }^{a}$ Hyun-Sung Lee, MD, PhD, ${ }^{a}$ Hee-Jin Jang, MD, ${ }^{\mathrm{a}}$ Jungnam Joo, PhD, ${ }^{\mathrm{b}}$ \\ Moon Soo Kim, MD, ${ }^{a}$ Jong Mog Lee, MD, ${ }^{a}$ and Jae Ill Zo, MD, PhD ${ }^{\mathrm{a}}$
}

Objectives: Sleeve lobectomy was introduced for patients with lung cancer whose pulmonary reserve was inadequate for pneumonectomy. However, the safety and survival benefits of wedge bronchoplastic lobectomy as an alternative to sleeve lobectomy have not been thoroughly studied. This study was performed to evaluate the safety and oncologic results of wedge bronchoplastic lobectomy for lung cancer.

Methods: We retrospectively analyzed 191 patients who underwent wedge bronchoplastic lobectomy and mediastinal lymph node dissection from 2001 to 2009.

Results: There were 174 male patients with a mean age of $61.8 \pm 8.2$ years. The median follow-up duration was 28 months. Nine patients showed severe postoperative complications: bronchopleural fistulas $(\mathrm{n}=3)$, necrosis at the bronchoplasty site $(n=1)$, or obstruction $(n=5)$. The operative mortality rate was $3.7 \%$. Local and regional recurrences were reported in 17 and 12 patients, respectively. The 5 -year overall survival was $62.8 \%$. The 5 -year overall survival was $68.6 \%$ in N0, $64.4 \%$ in N1, and $52.6 \%$ in N2 $(P=.09)$. The 5 -year overall freedoms from local recurrence and locoregional recurrence were $85.3 \%$ and $78.9 \%$, respectively, which did not differ by nodal status. A multivariate analysis showed that positive $\mathrm{N} 1$ and $\mathrm{N} 2$ nodes were risk factors $(P=.036$ and $P=.042$, respectively) for overall survival after wedge bronchoplastic lobectomy.

Conclusions: Wedge bronchoplastic lobectomy for lung cancer is a safe and feasible procedure that does not compromise oncologic principles. It can be considered an appropriate alternative to sleeve lobectomy and pneumonectomy, regardless of nodal status. (J Thorac Cardiovasc Surg 2012;143:825-31)

Earn CME credits at

http://cme.ctsnetjournals.org

Supplemental material is available online.

Bronchoplastic procedures were initially introduced as alternative procedures for patients with lung cancer whose pulmonary reserve was thought to be inadequate for tolerating pneumonectomy; it has now become the standard procedure, regardless of the patient's functional status. There are 3 types of bronchoplastic procedures: sleeve lobectomy, flap bronchoplasty, and wedge bronchoplasty. ${ }^{1}$ Several reports have shown that sleeve lobectomy has several

From the Center for Lung Cancer ${ }^{\mathrm{a}}$ and the Cancer Biostatistics Branch, ${ }^{\mathrm{b}}$ Research Institute and Hospital, National Cancer Center, Goyang, Gyeonggi, Republic of Korea.

Disclosures: Authors have nothing to disclose with regard to commercial support. Received for publication April 26, 2011; revisions received Sept 14, 2011; accepted for publication Oct 21, 2011; available ahead of print Nov 21, 2011.

Address for reprints: Hyun-Sung Lee, Center for Lung Cancer, Research Institute and

Hospital, National Cancer Center, 323 Ilsan-ro, Ilsandong-gu, Goyang, Gyeonggi,

410-769, Korea (E-mail: thoracic@ncc.re.kr).

$0022-5223 / \$ 36.00$

Copyright (c) 2012 by The American Association for Thoracic Surgery

doi:10.1016/j.jtcvs.2011.10.057 advantages over pneumonectomy in terms of operative morbidity, mortality, and long-term oncologic results. ${ }^{2-4}$ After sleeve lobectomy, however, the circumferential resection of the bronchus and the intraoperative interruption of the bronchial vascular supply to the distal bronchial segment can result in ischemia at the anastomosis site and subsequent devastating complications, such as bronchial strictures or bronchopleural fistulas (BPFs). ${ }^{5}$ Approximately $2.3 \%$ to $5 \%$ of patients have bronchial strictures after sleeve lobectomy and $2 \%$ to $6.9 \%$ of patients have BPFs. $^{6}$

Rather than circumferentially resecting the bronchus, wedge bronchoplastic lobectomy resects in a wedge shape and reanastomizes it. This procedure can preserve the blood supply to the distal bronchus and subsequently reduce the ischemia at the anastomotic site and prevent circumferential strictures. Krüger and associates ${ }^{7}$ reported a significantly higher rate of major anastomotic complications and pneumonias after sleeve lobectomy than after wedge bronchoplasty. Despite these merits, wedge bronchoplastic lobectomies have not been extensively performed by thoracic surgeons, in some cases because many surgeons think that both tumor and nodal clearance are less satisfactory with wedge bronchoplastic lobectomy than they are with sleeve lobectomy. ${ }^{5}$ Also, Khargi and coworkers ${ }^{1}$ have reported that anastomotic complications, such as bronchial stenosis, frequently developed after wedge bronchoplastic lobectomy. Furthermore, there have been few studies 


\section{Abbreviations and Acronyms \\ $\mathrm{BPF}=$ bronchopleural fistula \\ $\mathrm{BVF}=$ bronchovascular fistula \\ $\mathrm{CI}=$ confidence interval \\ CIS = carcinoma in situ \\ NSCLC $=$ non-small cell lung cancer}

examining the long-term oncologic and survival outcomes of wedge bronchoplastic lobectomies. ${ }^{1,8}$

At our institution, wedge bronchoplastic lobectomy has been performed as an alternative procedure to sleeve lobectomy in cases of non-small cell lung cancer (NSCLC) involving or protruding into the main bronchus. Therefore, our experience provides a unique opportunity to evaluate the procedure's safety and long-term survival, focusing on locoregional recurrences. This evaluation was the main purpose of our study.

\section{METHODS \\ Patients}

This retrospective study was approved by the institutional review board of the hospital (IRB No. NCCNCS-10-419). We retrospectively reviewed medical and surgical records and bronchoscopic findings. One hundred ninety-one patients who underwent wedge bronchoplastic lobectomies with complete mediastinal lymph dissection for NSCLC between March 2001 and January 2009 were enrolled. All tumors invading or protruding at the orifice of the main bronchus were indicated for wedge bronchoplasty lobectomy. During the same period, 43 cases of sleeve lobectomy were performed at the same institution (Figure E1). In $14(32.5 \%)$ of 43 cases, however, wedge bronchoplastic lobectomy was considered first, but we eventually had to perform sleeve lobectomy because a sufficient resection margin could not be obtained by wedge bronchoplasty, despite multiple frozen sections.

The general characteristics of the patients are shown in Table 1. The median follow-up period was 28 months (range, $0.8-91.5$ months). Chest computed tomography, positron emission tomography, bronchoscopy, and pulmonary function testing were performed preoperatively. Bronchoscopic inspection has been routinely performed on postoperative day 7 since 2006 . Until 2005, a bronchoscopic inspection was performed when surgical morbidities, such as stenosis, fistulas, or distortions, were clinically suspected. Adjuvant chemotherapy was considered in patients with nodal metastasis and adjuvant radiotherapy was considered when the resection margin was above the dysplasia on the permanent pathologic report and the patient could medically tolerate it. Postoperative chest computed tomographic scans were obtained at 3-month intervals, and positron emission tomographic scans were obtained annually to detect recurrences.

Local recurrences were defined as those occurring on the bronchial resection margins and airways, such as the bronchial stumps, bronchi, carina, or trachea. Regional recurrences were defined as those occurring in the hilar or mediastinal lymph nodes and the pleural cavity. Distant recurrences were defined as those occurring in the lung, brain, liver, adrenal glands, bones, and other locations. An R1 resection of the bronchial margin was defined as carcinoma in situ (CIS) or invasive carcinoma at the bronchial margin. ${ }^{9}$

\section{Surgical Technique}

The indication for wedge bronchoplastic lobectomy was the presence on preoperative bronchoscopy of endobronchial lesions that had invaded or
TABLE 1. General characteristics of the patients

\begin{tabular}{|c|c|}
\hline Variables & No. $(\%)$ \\
\hline Age (y) & $61.8 \pm 8.2$ \\
\hline Gender (male/female) & $174(91.9 \%) / 17(8.9 \%)$ \\
\hline Preoperative chemotherapy & $24(12.6 \%)$ \\
\hline \multicolumn{2}{|l|}{ Location of lesion } \\
\hline RUL & $93(48.7 \%)$ \\
\hline RML & $14(7.3 \%)$ \\
\hline RLL & $25(13.1 \%)$ \\
\hline RUL + RML & $3(1.6 \%)$ \\
\hline RML + RLL & $1(0.5 \%)$ \\
\hline LUL & $37(19.4 \%)$ \\
\hline LLL & $18(9.4 \%)$ \\
\hline \multicolumn{2}{|l|}{ Nodal status } \\
\hline No & $67(35.1 \%)$ \\
\hline N1 & $81(42.4 \%)$ \\
\hline $\mathrm{N} 2$ & $43(22.5 \%)$ \\
\hline \multicolumn{2}{|l|}{ Pathologic stage* } \\
\hline I & $40(20.9 \%)$ \\
\hline II & $80(41.9 \%)$ \\
\hline III & $71(37.2 \%)$ \\
\hline \multicolumn{2}{|l|}{ Pathologic cell type } \\
\hline Squamous cell carcinoma & $153(80.1 \%)$ \\
\hline Adenocarcinoma & $21(10.9 \%)$ \\
\hline Others & $17(9.0 \%)$ \\
\hline Pulmonary angioplasty & $16(8.4 \%)$ \\
\hline Operative time (min) & $232.9 \pm 69.5$ \\
\hline Hospital days (d) & $14.6 \pm 10.5$ \\
\hline Operative mortality & $7(3.7 \%)$ \\
\hline Postoperative chemotherapy & $66(34.6 \%)$ \\
\hline Postoperative radiotherapy & $15(7.9 \%)$ \\
\hline
\end{tabular}

$R U L$, Right upper lobe; $R M L$, right middle lobe; $R L L$, right lower lobe; $L U L$, left upper lobe; $L L L$, left lower lobe. *American Joint Committee on Cancer 2010 lung cancer stage.

protruded into the main bronchus; therefore, conventional stapling devices could not be applied for the bronchial resection. The lungs were ventilated with a double-lumen tube in all patients. A standard posterolateral thoracotomy was performed in the lateral decubitus position. The pleural space was entered through the fifth intercostal space. After dissection of the intrapulmonary ligament, the pulmonary vein and lobar pulmonary arteries were transected, and a complete mediastinal lymph node dissection was performed. The systemic blood supply via the bronchial artery was preserved as much as possible. The tumor was palpated, and a bronchotomy was performed to assess the precise intraluminal tumor location. The affected lung lobe and a portion of the main bronchus were excised through 2 oblique bronchial incisions in the main bronchus (a wedge resection), in contrast to circumferential resection of the bronchus in sleeve lobectomy (Figure 1,A). In this way, over half the circumference of the main bronchus was excised and trimmed. After the negative resection margins had been confirmed by frozen pathologic examination, the bronchial edges were approximated or plicated. The cartilaginous part was sutured with interrupted or figure-of-8 4-0 PDS polydioxanone sutures (Ethicon, Inc, Somerville, $\mathrm{NJ})$. After the anastomosis was completed, an intraoperative bronchoscopic inspection was performed to evaluate it. The anastomosis was covered with pedicled pericardial fat or a pleural flap in all the patients. In some cases, pericardial release was performed to reduce the tension on the suture line. A schematic illustration of a wedge bronchoplastic lobectomy is shown in Figure 1, $B$. Figure 1, $C$, shows a 3-dimensional computed tomographic reconstruction image after a wedge bronchoplastic right upper lobectomy, with the remainder of the bronchus folded outside. 

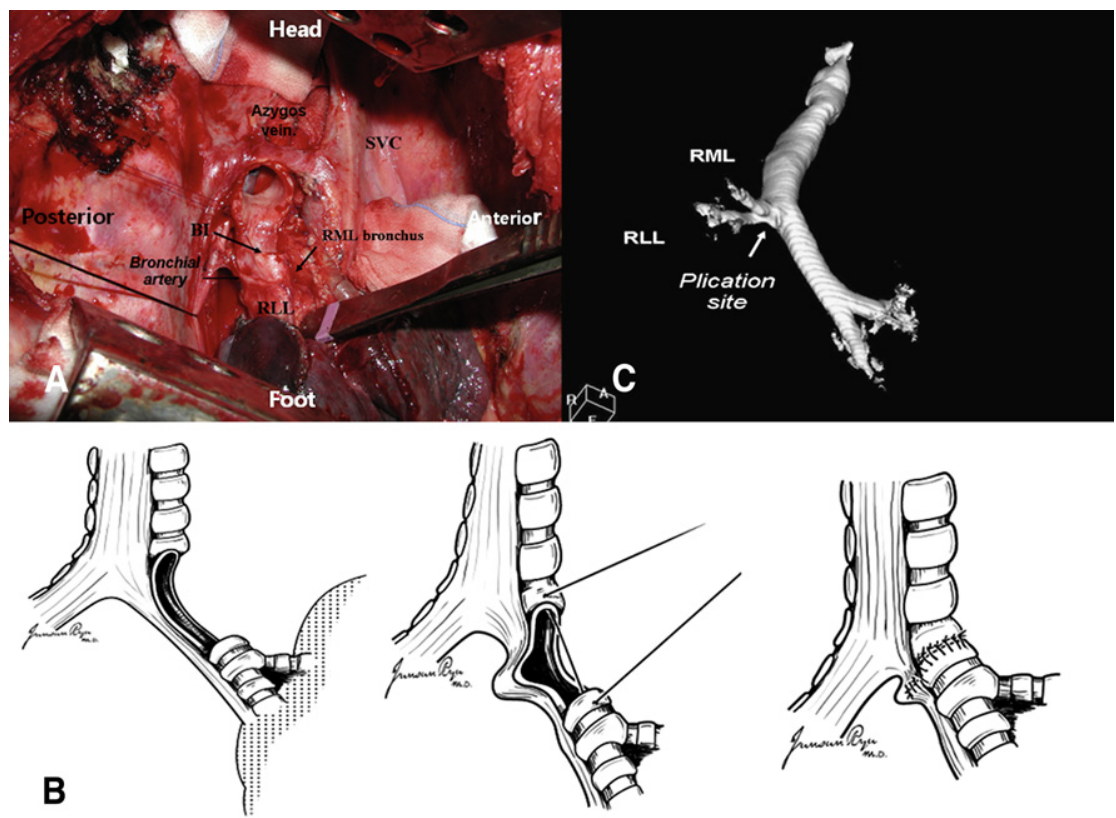

FIGURE 1. An extensive wedge bronchoplastic lobectomy. A, The intraoperative findings for a wedge bronchoplastic right upper lobectomy. The right upper lobe bronchus and bronchus intermedius were resected into a wedge shape. B, A schematic illustration of a wedge bronchoplastic lobectomy. After the wedge resection of the bronchus, the right upper lobe was removed, and the remnant bronchus was closed. To prevent folding of the remainder of the bronchus into the lumen of the bronchus, additional sutures can be added to the remainder of bronchus. C, A 3-dimensional reconstruction image after a wedge bronchoplastic right upper lobectomy. The remainder of the bronchus (arrow at the plication site) was folded outside the lumen of the bronchus. In this case, the folded bronchus did not result in narrowing of the bronchial lumen. $S V C$, Superior vena cava; $R M L$, right middle lobe; $R L L$, right lower lobe; $B I$, bronchus intermedius.

\section{Bronchoscopic Findings}

The findings of the bronchoscopic inspection on postoperative day 7 were classified into 4 categories of complications: normal, mild, moderate, and severe (Figure E2). Mild complications were defined as erythema or edema, without luminal narrowing at the bronchoplasty site. After the resection of the bronchus and anastomosis, the remainder of the bronchus could, in some cases, fold into or outside the lumen of the bronchus. An "inner-fold" was defined as having this inner folding into the lumen. Moderate complications were defined as this inner folding or as mild ischemia with mild luminal narrowing but without narrowing-related complications, such as obstructive pneumonia or dyspnea. Severe complications were defined as severe ischemia, BPFs, total obstruction, or necrosis at the bronchoplasty site.

\section{Statistical Analysis}

Statistical analysis was performed using STATA 11 software (Stata Corp, Stata Statistical Software, Release 11 [2005], College Station, Tex). Patient survival was analyzed using the date of the procedure as the starting point, and the Kaplan-Meier method was used to estimate the survival. The log-rank test was used to compare the survival in the univariate analysis. A Cox proportional hazard model with backward variable selection at a significance level of .05 was used for the multivariate analysis.

\section{RESULTS}

\section{Pathologic Results of Resection Margin}

In the frozen pathologic reports, 184 patients had negative margins, $4(2.1 \%)$ patients had dysplasia, and 3 $(1.6 \%)$ patients had CIS. In the permanent pathologic reports, however, $175(91.1 \%)$ patients showed a negative resection margin. Two $(1.0 \%)$ patients were reported as having metaplasia, and $6(3.1 \%)$ patients were reported as having dysplasia. An R1 resection was reported in 8 $(4.2 \%)$ patients: $7(3.7 \%)$ with CIS and $1(0.5 \%)$ with invasive carcinoma. Of these 8 patients, 5 had a diagnosis of negative resection margins, 1 had a diagnosis of dysplasia, and 2 had a diagnosis of CIS on the frozen pathologic report (Table E1).

Of the 14 patients given a diagnosis of dysplasia, CIS, or invasive carcinoma, 2 had local recurrence and 5 had distant metastasis. Postoperative radiotherapy was performed in 3 of these 14 patients, and they did not have local recurrence. The outcomes for these patients are summarized in Figure E3.

\section{Bronchoscopic Follow-up After Wedge Bronchoplastic Lobectomy}

A postoperative bronchoscopic follow-up was performed in $95(49.7 \%)$ patients. The 96 patients who did not undergo bronchoscopic follow-ups showed no clinical evidence of complications. Among the 95 patients with bronchoscopic follow-up who did undergo follow-up, 37 patients had normal bronchoscopic findings. Overall, 15 patients experienced mild complications, 34 had moderate complications, and 9 patients had severe complications, such as BPFs 
$(\mathrm{n}=3)$, necrosis at the bronchoplasty site $(\mathrm{n}=1)$, or severe ischemia $(\mathrm{n}=5)$. The severe ischemic lesions at the bronchoplasty site eventually led to total obstruction on the follow-up bronchoscopy.

\section{Surgical Morbidities and Mortalities}

Of the common complications of wedge bronchoplastic lobectomy, sputum retention requiring bronchoscopic toileting and atelectasis developed in 28 (14.7\%) patients. Lung injuries, such as acute lung injuries or acute respiratory distress syndrome, developed in $8(4.2 \%)$ patients. Of the 3 patients who had BPFs, 2 recovered with conservative treatment, and 1 underwent the Eloesser procedure owing to BPF-related empyema. Necrosis at the bronchoplasty site developed in 1 patient, and he underwent completion pneumonectomy owing to superimposed fungal infection. Five $(2.6 \%)$ patients had significant narrowing and near total obstruction at the bronchoplasty site. Of them, 2 patients received repeated bronchoscopic balloon dilatation with argon plasma coagulation therapy. Three patients did not receive further treatment for narrowing or obstruction because they did not have clinical symptoms, such as dyspnea or obstructive pneumonia. Bronchovascular fistulas (BVFs) did not develop in this patient series. The operative mortality rate was $3.7 \%$ (7 cases), with the following causes: acute myocardial infarction $(\mathrm{n}=1)$, postoperative pneumonia $(\mathrm{n}=1)$, and acute respiratory distress syndrome $(n=5)$. The mortality case of the postoperative pneumonia was related to $\mathrm{BPF}$.

\section{Patterns of Recurrence and Survival}

Recurrence was reported in 59 patients, with 17 local recurrences (10 in the stump and 7 in the trachea or carina), 12 regional recurrences ( 7 pleural or chest wall masses and 5 mediastinal lymph nodes), and 43 distant recurrences (17 lung, 8 brain, 6 liver, 7 bone, 2 adrenal glands, and 10 other). Seven patients had multiple distant recurrences. Four patients had both local and distant recurrences, and 9 patients had both regional and distant recurrences. Two patients had double primary lung cancer after the operation.

The 5-year overall survival after wedge bronchoplastic lobectomy was $62.8 \%$ (95\% confidence interval [CI], $49.9 \% \sim 73.2 \%$ ), and the 5-year disease-free survival was $50.0 \% \quad(95 \%$ CI, 40.4\% 58.8\%). According to the Kaplan-Meier analysis, the 5-year overall survival was $68.6 \%(95 \%$ CI, 38.5\% 86.1\%) in the N0 cases, $64.4 \%$ $(95 \% \mathrm{CI}, 49.8 \% \sim 75.7 \%)$ in the $\mathrm{N} 1$ cases, and $52.6 \%$ $(95 \% \mathrm{CI}, 26.9 \% \sim 73.0 \%)$ in the $\mathrm{N} 2$ cases $(P=.09$ for the log-rank test; Figure 2, A). The 5-year overall survival was $60.6 \%$ (95\% CI, 46.6\% 71.9\%) in the patients whose resection margins were negative or who had metaplasia and $84.6 \%(95 \% \mathrm{CI}, 50.4 \% \sim 95.9 \%)$ in the patients with
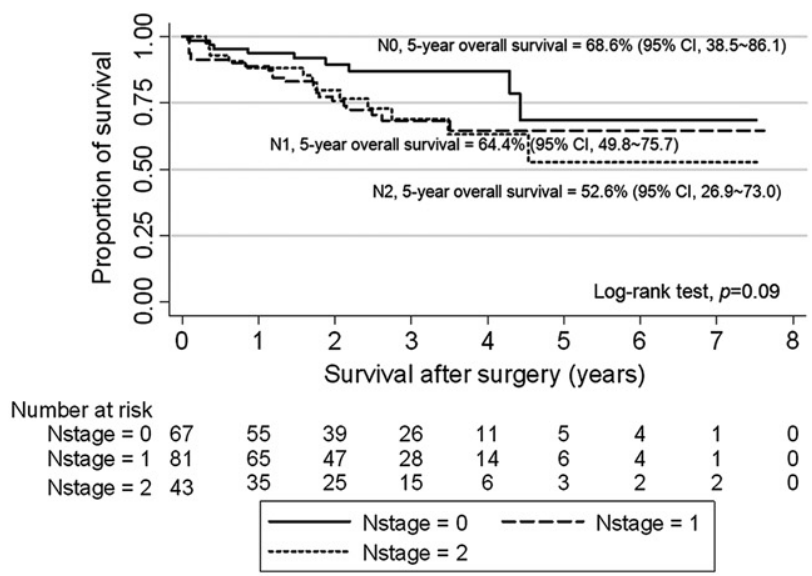

A Overall survival according to nodal status

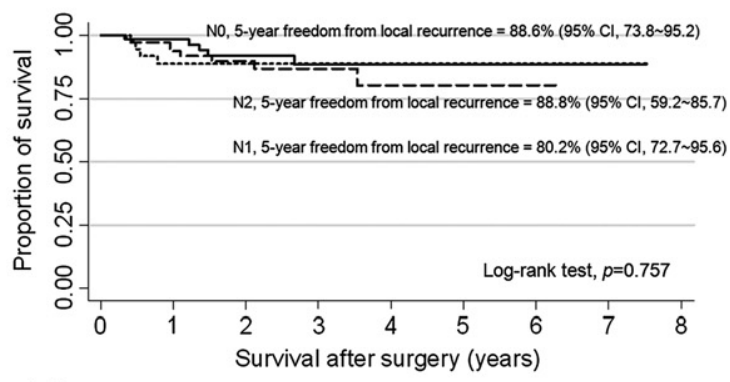

Number at risk

$\begin{array}{llllllllll}\text { Nstage }=0 & 67 & 51 & 32 & 22 & 10 & 5 & 4 & 1 & 0 \\ \text { Nstage }=1 & 81 & 52 & 34 & 22 & 10 & 5 & 3 & 0 & 0\end{array}$

Nstage $=1 \quad 8$

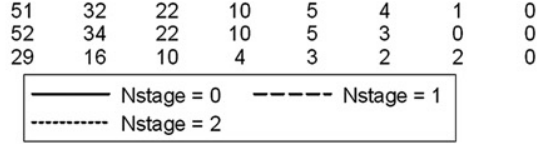

B Freedom from local recurrence according to nodal status
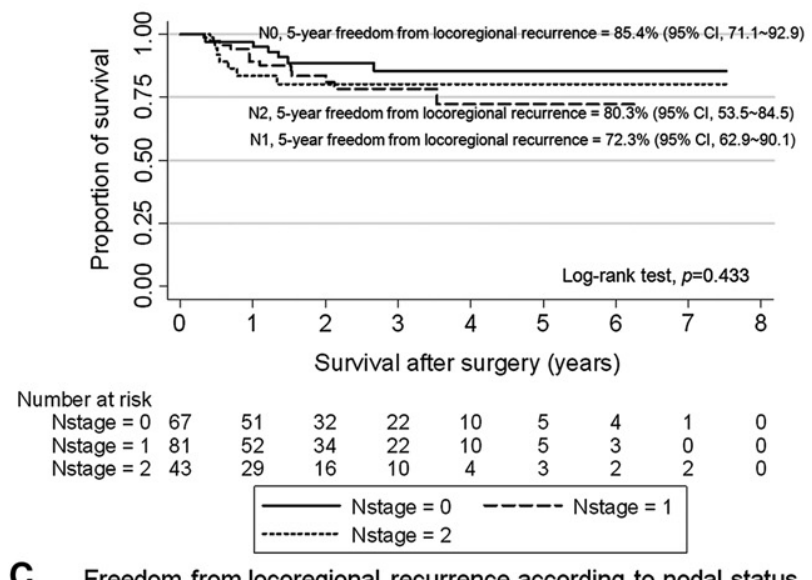

FIGURE 2. Survival curves. A, Overall survival according to nodal status. B, Freedom from local recurrence according to nodal status. C, Freedom from locoregional recurrence according to nodal status. CI, Confidence intervals.

dysplasia, CIS, or carcinoma $(P=.342$ for the log-rank test).

The 5-year overall rate for freedom from local recurrence was $85.3 \%(95 \% \mathrm{CI}, 75.8 \% \sim 91.2 \%)$, and the 5-year 
TABLE 2. The risk factors for overall survival after wedge bronchoplastic lobectomy using a Cox proportional hazard model

\begin{tabular}{|c|c|c|c|c|}
\hline & \multicolumn{2}{|c|}{ Univariate analysis } & \multicolumn{2}{|c|}{ Multivariate analysis } \\
\hline & HR $(95 \%$ CI $)$ & $P$ value & HR $(95 \%$ CI $)$ & $P$ value \\
\hline Age & $1.039(0.998-1.082)$ & .062 & - & - \\
\hline Gender (male vs female) & $2.112(0.511-8.727)$ & .302 & - & - \\
\hline Preoperative chemotherapy & $1.628(0.723-3.662)$ & .239 & - & - \\
\hline \multicolumn{5}{|l|}{ T stage } \\
\hline $\mathrm{T} 2$ (vs T1) & $1.802(0.777-4.176)$ & .170 & - & - \\
\hline $\mathrm{T} 3$ (vs T1) & $1.630(0.602-4.417)$ & .337 & - & - \\
\hline $\mathrm{T} 4$ (vs T1) & $2.256(0.658-7.732)$ & .196 & - & - \\
\hline \multicolumn{5}{|l|}{ Nodal status ( $\mathrm{N}$ stage) } \\
\hline N1 (vs N0) & $2.203(1.019-4.763)$ & .045 & $2.311(1.057 \sim 5.051)$ & .036 \\
\hline N2 (vs N0) & $2.241(0.958-5.244)$ & .063 & $2.426(1.032 \sim 5.701)$ & .042 \\
\hline Resection margins (above the dysplasia) & $0.509(0.123-2.105)$ & .351 & - & - \\
\hline Concomitant pulmonary angioplasty & $1.710(0.724-4.042)$ & .221 & - & - \\
\hline Severe bronchial complications & $0.622(0.085-4.531)$ & .639 & - & - \\
\hline Postoperative chemotherapy & $1.600(0.884-2.897)$ & .120 & $1.778(0.978 \sim 3.233)$ & .059 \\
\hline Postoperative radiotherapy & $0.606(0.226-2.377)$ & .734 & - & - \\
\hline
\end{tabular}

Only the variables that were selected via backward variable selection at a significance threshold of 0.05 in the multivariate analysis are listed. $H R$, Hazard ratio; $C I$, confidence intervals.

overall freedom from locoregional recurrence was 78.9\% $(95 \%$ CI, $69.6 \% \sim 85.7 \%)$. The 5-year overall freedom from local recurrence was $88.6 \%(95 \%$ CI, $73.8 \%$ $95.2 \%)$ in the N0 cases, $80.2 \%(95 \%$ CI, $59.2 \%$ $91.1 \%)$ in the $\mathrm{N} 1$ cases, and $88.8 \%(95 \% \mathrm{CI}, 72.7 \%$ $95.6 \%)$ in the N2 cases $(P=.757$; Figure $2, B)$. The 5 year freedom from locoregional recurrence was $85.4 \%$ (95\% CI, $71.1 \% \sim 92.9 \%)$ in the N0 cases, $72.3 \%(95 \%$ CI, $53.5 \% \sim 92.9 \%)$ in the N1 cases, and $80.3 \%(95 \%$ CI, $62.9 \% \sim 90.1 \%)$ in the N2 cases $(P=.433$; Figure 2, $C)$. There were no significant differences in freedom from local and locoregional recurrence based on nodal status.

\section{Risk Factors for Overall Survival After Wedge Bronchoplastic Lobectomy}

Age, sex, preoperative chemotherapy, $\mathrm{T}$ stage, $\mathrm{N}$ stage (nodal status), resection margins (dysplasia, CIS, and carcinoma vs negative for cancer and metaplasia in the permanent resection margins), concomitant pulmonary angioplasty, severe postoperative complications, postoperative chemotherapy, and radiotherapy were included in using the Cox proportional hazards model (Table 2). In the univariate analysis, N1 was a significant risk factor when compared with N0 $(P=.045)$, and age $(P=.062)$ and N2, compared with $\mathrm{N} 0(P=.063)$, were marginally significant. In the multivariate analysis, $\mathrm{N} 1(P=.036)$ and $\mathrm{N} 2$ $(P=.042)$ compared with N0 were significant risk factors for overall survival after wedge bronchoplastic lobectomy. Positive resection margins and concomitant pulmonary angioplasty were not related to overall survival in this analysis.

\section{DISCUSSION}

This study demonstrates that wedge bronchoplastic lobectomy for lung cancer has acceptable complications and oncologic results. The incidence of local and regional recurrence is at the center of the debate regarding the long-term results of sleeve lobectomy versus pneumonectomy. Local disease control is one of the main goals of surgical resection for lung cancer. ${ }^{10}$ Some surgeons believe that if the tumor cells involve the peribronchial lymphatics in $\mathrm{N} 1$ or N2 disease, then sleeve lobectomy may increase the incidence of locoregional recurrence. ${ }^{2}$ However, many studies have already concluded that sleeve lobectomy does not increase the incidence of local and regional recurrence compared with pneumonectomy; therefore, sleeve lobectomy should be performed instead of pneumonectomy in patients with NSCLC, regardless of their nodal status, whenever complete resection can be achieved because it is a lung-saving procedure with lower postoperative risks and is as curative as pneumonectomy. ${ }^{4}$ In cases of wedge bronchoplastic lobectomy, local and regional recurrences are also matters of primary consideration. Most surgeons believe that both tumor and nodal clearance are less satisfactory with wedge bronchoplastic lobectomy than they are with sleeve lobectomy; therefore, they assume that the incidence of local and locoregional recurrence in wedge bronchoplastic lobectomy is greater than that of sleeve lobectomy. ${ }^{5}$ In this study, however, the incidence of local recurrence was $8.9 \%$ in wedge bronchoplasty, which is comparable with the reported data for sleeve lobectomy $(5 \%-24 \%){ }^{11,12}$ If the definition of local recurrence is limited to stump recurrence, then the incidence of local recurrence was only $5.2 \%$. Regarding long-term survival, our results of wedge bronchoplastic lobectomy were comparable with previous reports of sleeve lobectomy (Table 3). Even in the $\mathrm{N} 2$ cases, the 5-year overall survival was greater than $50 \%$. In addition, freedom from local and locoregional recurrence did not differ by nodal status. These results imply 
TABLE 3. Comparison of outcome between current study of wedge bronchoplastic lobectomy and previous studies of sleeve lobectomies

\begin{tabular}{|c|c|c|c|c|c|c|c|c|c|c|c|}
\hline \multirow[b]{2}{*}{ First author } & \multirow[b]{2}{*}{ Year } & \multirow{2}{*}{$\begin{array}{c}\begin{array}{c}\text { No. of } \\
\text { patients }\end{array} \\
\end{array}$} & \multirow{2}{*}{$\begin{array}{c}\begin{array}{c}\text { Bronchial } \\
\text { stenosis }(\%)\end{array} \\
\end{array}$} & \multirow{2}{*}{$\begin{array}{l}\text { BPF } \\
(\%) \\
\end{array}$} & \multirow{2}{*}{$\begin{array}{c}\text { Operative } \\
\text { mortality }(\%)\end{array}$} & \multicolumn{4}{|c|}{ 5-yr overall survival $(\%)$} & \multirow{2}{*}{$\begin{array}{c}\text { Local } \\
\text { recurrence }(\%)\end{array}$} & \multirow{2}{*}{$\begin{array}{c}\mathbf{R} 1 \\
\text { resection }(\%)\end{array}$} \\
\hline & & & & & & All & No & N1 & $\mathbf{N 2}$ & & \\
\hline Tedder $^{6}$ & 1992 & 1915 & 5.0 & 3.5 & 7.5 & - & - & - & - & 10.3 & - \\
\hline Van Schill $^{15}$ & 1996 & 145 & - & - & 4.8 & 46 & 62 & 29 & 31 & 20 & - \\
\hline $\operatorname{Rea}^{14}$ & 1997 & 217 & 2.3 & 2.3 & 6.2 & 49 & 72 & 36 & 22 & 8.7 & - \\
\hline Deslauriers $^{5}$ & 2004 & 300 & - & - & 2.7 & 54 & 66 & 50 & 19 & 16 & - \\
\hline $\mathrm{Kim}^{12}$ & 2005 & 49 & 4.0 & 2.0 & 6.1 & 53.7 & 74.8 & 51.5 & - & 24 & 6 \\
\hline Ludwig $^{3}$ & 2005 & 116 & - & 6.9 & 4.3 & 39 & 56 & 38 & 24 & - & - \\
\hline This study & 2011 & 191 & 2.6 & 1.6 & 3.7 & 62.8 & 68.6 & 64.4 & 52.6 & 8.9 & 4.2 \\
\hline
\end{tabular}

$B P F$, Bronchopleural fistula.

that both the tumor and the nodal clearance of wedge bronchoplastic lobectomy are satisfactory. Therefore, wedge bronchoplastic lobectomy can be performed, regardless of the nodal status, without compromising oncologic principles. If the complete dissection of suspicious positive lymph nodes is possible, then nodal status is not a contraindication for wedge bronchoplastic lobectomy.

Concerning the resection margins after wedge bronchoplastic lobectomy, inconsistency between the frozen and permanent sections was inevitable. In cases of incomplete resection, however, other treatment modalities, such as radiotherapy or photodynamic therapy, could be considered after the operation. Also, regular and preemptive followup by bronchoscopy is important for detecting early local recurrence. Even if local recurrence is detected, a second procedure or local treatments could be treatment options. Furthermore, even when there is dysplasia or CIS in the resection margins, not all patients have local recurrence. Wind and colleagues ${ }^{9}$ have reported the results of a metaanalysis in which they concluded that the survival of patients with CIS at the bronchial resection margin was comparable with the survival after a radical resection.

Regarding the surgical mortality, the operative mortality rate for wedge bronchoplastic lobectomy in our study was $3.7 \%$. These results are similar to the previously reported operative mortalities for sleeve lobectomy $(2.7 \%-7.5 \%)$ (Table 3). ${ }^{13-16}$ In terms of surgical morbidity, wedge bronchoplastic lobectomy is also a safer procedure than sleeve lobectomy. The incidence of BPFs after sleeve lobectomy has been reported to be approximately $5 \%,{ }^{7}$ the incidence of BVFs has been reported to be from $0.9 \%$ to $3.2 \%$, and the incidence of late anastomotic strictures has been reported to be from $2.3 \%$ to $5 \% .^{5,14,17}$ Compared with these previous sleeve lobectomy results, the incidences of these common complications after wedge bronchoplastic lobectomy are relatively low. Nazari and coworkers ${ }^{18}$ assumed a correlation between a better systemic blood supply to the distal portions of the bronchus after bronchial wedge resection and better postoperative outcomes. In addition, BVFs, which are the most devastating complication in sleeve and bronchoplastic lobectomy, did not develop in this study. We believe that both the preserved blood supply to the distal bronchus and the pericardial or pleural flap that covered the bronchoplasty site played roles in preventing BVFs. Moreover, 2 of the 3 patients with BPFs recovered with only conservative treatment. If a pericardial or pleural flap covers the bronchoplasty site completely, any BPFs that occur can heal through granulation tissues without surgical intervention.

Similar to sleeve lobectomy, however, several anastomotic complications can also develop after wedge bronchoplastic lobectomy. As described earlier, mild or moderate ischemia can develop after wedge bronchoplastic lobectomy. In the process of healing from ischemia, granulation tissues can grow at the bronchoplasty site, and these granulation tissues can result in luminal narrowing. However, luminal narrowing without near total obstruction is not clinically significant. In contrast to circumferential strictures after sleeve lobectomy, luminal narrowing after wedge bronchoplastic lobectomy is not circumferential. The patency of the lumen can be preserved owing to the viable remnant bronchus, even if luminal narrowing has developed. However, inner folding is one of the important surgical complications of performing a wedge bronchoplastic lobectomy. This inner folding develops only after bronchoplasty and finally results in luminal narrowing. To prevent the "inner fold," we create a wide wedge resection and suture the edge of the bronchus obliquely. In some cases, we add extra sutures to the remainder of the bronchus to make an "outer fold" or to do the plication (Figure 1,B).

\section{Limitations}

This study had several limitations. The postoperative bronchoscopic follow-up inspections were performed in only half of the patients. Thus, the incidence of abnormal bronchoscopic findings may have been underestimated. However, we routinely performed intraoperative bronchoscopic evaluations after the anastomosis. If severe anastomotic complications (such as BPFs or bronchial stenosis) developed, they could be detected from the clinical symptoms. Therefore, we believe that the rate of severe anastomotic complications in this article probably reflects the clinical outcomes of wedge bronchoplastic lobectomy accurately. Second, we did not compare the surgical outcomes of 
wedge bronchoplastic lobectomy with outcomes of sleeve lobectomy. Because this was a retrospective study, the tumor characteristics of the sleeve lobectomy may have been more aggressive than those of the wedge bronchoplastic lobectomy. The 5-year overall survival and 5-year freedom from locoregional recurrence between the wedge bronchoplasty and sleeve lobectomy in our institute were added in Figure E4. However, because of the small number of sleeve lobectomy cases, the question of intent-to-treat, and the lack of centrality of this comparison to this study, we tried to emphasize the oncologic feasibility of wedge bronchoplastic lobectomy for primary lung cancer.

\section{CONCLUSIONS}

Wedge bronchoplastic lobectomy for lung cancer shows acceptable postoperative outcomes without compromising the oncologic principles. It can be performed regardless of nodal status and could be considered an appropriate alternative to sleeve lobectomy.

We are indebted to Dr Joon Seon Ryu at the National Cancer Center for the illustrations.

\section{References}

1. Khargi K, Duurkens VA, Versteegh MM, Huysmans HA, Quanjer PH, Verzijlbergen FF, et al. Pulmonary function and postoperative complications after wedge and flap reconstructions of the main bronchus. $J$ Thorac Cardiovasc Surg. 1996;112:117-23.

2. Deslauriers J, Grégoire J, Jacques LF, Piraux M, Guojin L, Lacasse Y, et al. Sleeve lobectomy versus pneumonectomy for lung cancer: a comparative analysis of survival and sites or recurrences. Ann Thorac Surg. 2004;77:1152-6.

3. Ludwig C, Stoelben E, Olschewski M, Hasse J. Comparison of morbidity, 30-day mortality, and long-term survival after pneumonectomy and sleeve lobectomy for non-small cell lung carcinoma. Ann Thorac Surg. 2005;79:968-73.

4. Okada M, Yamagishi H, Satake S, Matsuoka H, Miyamoto Y, Yoshimura M, et al. Survival related to lymph node involvement in lung cancer after sleeve lobec- tomy compared with pneumonectomy. J Thorac Cardiovasc Surg. 2000;119: 814-9.

5. Deslauriers J, Tronc F, Grégoire J. History and current status of bronchoplastic surgery for lung cancer. Gen Thorac Cardiovasc Surg. 2009;57:3-9.

6. Tedder M, Anstadt MP, Tedder SD, Lowe JE. Current morbidity, mortality, and survival after bronchoplastic procedures for malignancy. Ann Thorac Surg. 1992; 54:387-91.

7. Krüger M, Uschinsky K, Hässler K, Engelmann C. Postoperative complications after bronchoplastic procedures in the treatment of bronchial malignancies. Eur J Cardiothorac Surg. 1998;14:46-53.

8. Kotoulas C, Lazopoulos G, Foroulis C, Konstantinou M, Tomos P, Lioulias A. Wedge resection of the bronchus: an alternative bronchoplastic technique for preservation of lung tissue. Eur J Cardiothorac Surg. 2001;20:679-83.

9. Wind J, Smit EJ, Senan S, Eerenberg JP. Residual disease at the bronchial stump after curative resection for lung cancer. Eur $J$ Cardiothorac Surg. 2007;32:29-34.

10. Massard G, Kessler R, Gasser B, Ducrocq X, Elia S, Gouzou S, et al. Local control of disease and survival following bronchoplastic lobectomy for non-small cell lung cancer. Eur J Cardiothorac Surg. 1999;16:276-82.

11. Bagan P, Berna P, Pereira JC, Le Pimpec Barthes F, Foucault C, Dujon A, et al. Sleeve lobectomy versus pneumonectomy: tumor characteristics and comparative analysis of feasibility and results. Ann Thorac Surg. 2005;80:2046-50.

12. Kim YT, Kang CH, Sung SW, Kim JH. Local control of disease related to lymph node involvement in non-small cell lung cancer after sleeve lobectomy compared with pneumonectomy. Ann Thorac Surg. 2005;79:1153-61.

13. Nagayasu T, Matsumoto K, Tagawa T, Nakamura A, Yamasaki N, Nanashima A. Factors affecting survival after bronchoplasty and broncho-angioplasty for lung cancer: single institutional review of 147 patients. Eur J Cardiothorac Surg. 2006;29:585-90.

14. Rea F, Loy M, Bortolotti L, Feltracco P, Fiore D, Sartori F. Morbidity, mortality, and survival after bronchoplastic procedures for lung cancer. Eur J Cardiothorac Surg. 1997;11:201-5.

15. Van Schil PE, Brutel de la Riviére A, Knaepen PJ, van Swieten HA, Reher SW, Goossens DJ, et al. Long-term survival after bronchial sleeve resection: univariate and multivariate analyses. Ann Thorac Surg. 1996;61:1087-91.

16. Watanabe Y, Shimizu J, Oda M, Hayashi Y, Watanabe S, Yazaki U, et al. Results in 104 patients undergoing bronchoplastic procedures for bronchial lesions. Ann Thorac Surg. 1990;50:607-14.

17. Icard P, Regnard JF, Guibert L, Magdeleinat P, Jauffret B, Levasseur P. Survival and prognostic factors in patients undergoing parenchymal saving bronchoplastic operation for primary lung cancer: a series of 110 consecutive cases. Eur J Cardiothorac Surg. 1999;15:426-32.

18. Nazari S, Nascimbene G, Mourad Z, Fraipont G. Invaginated bronchoplasty: wedge resection and sleeve reconstruction. Minerva Chir. 1996;51:413-9. 


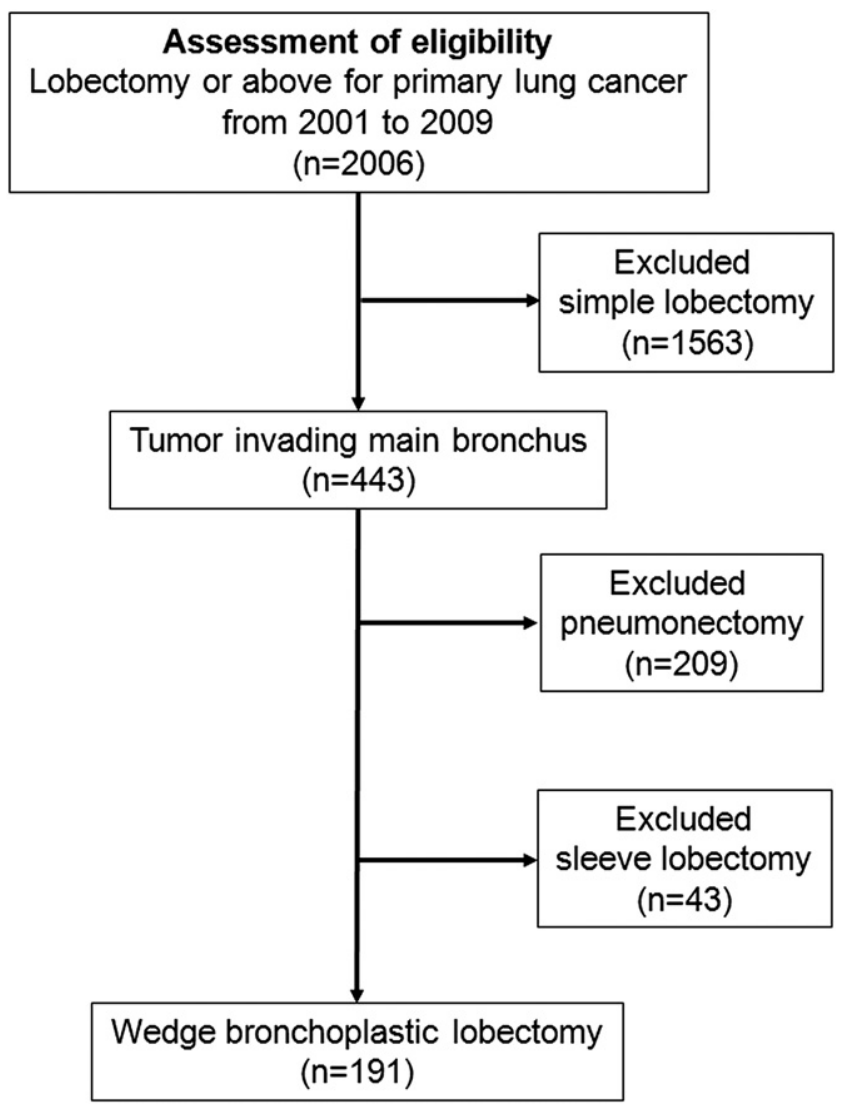

FIGURE E1. CONSORT diagram of the patients in this study. 


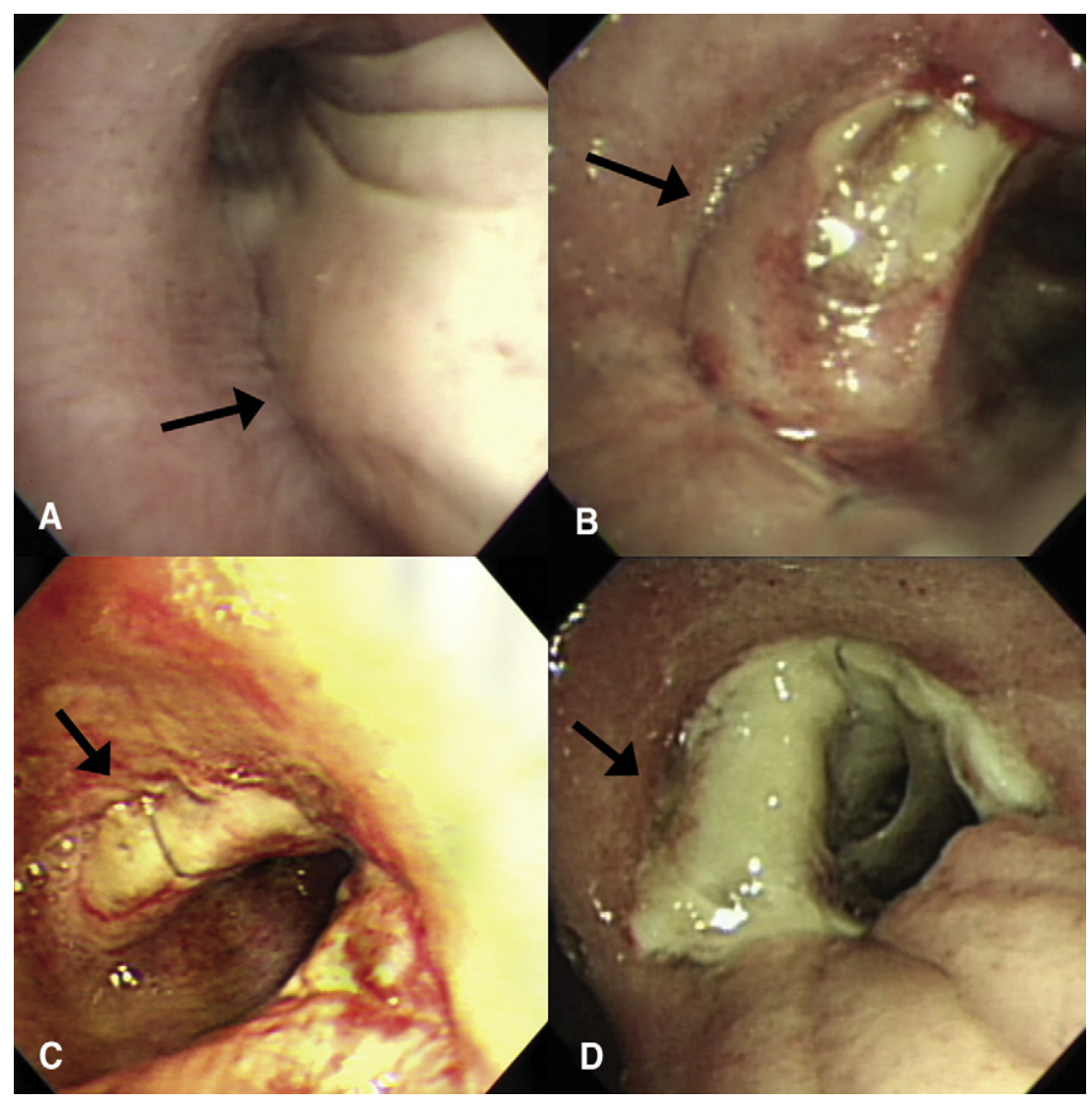

FIGURE E2. The postoperative bronchoscopic findings. A, A normal finding after wedge bronchoplastic lobectomy. B, Mild ischemia at the bronchoplasty site without luminal narrowing after a wedge bronchoplastic right upper lobectomy. C, Moderate ischemia and luminal narrowing at the bronchoplasty site after a wedge bronchoplastic left upper lobectomy. In contrast to the circumferential stricture after a sleeve lobectomy, the remnant portion of the bronchus is viable, and patency is preserved. D, Severe ischemia and necrosis at the bronchoplasty site after left upper bronchoplastic lobectomy. This lesion gradually became pinpoint, and the patient eventually received balloon dilatation. (Arrow = bronchoplasty site.)

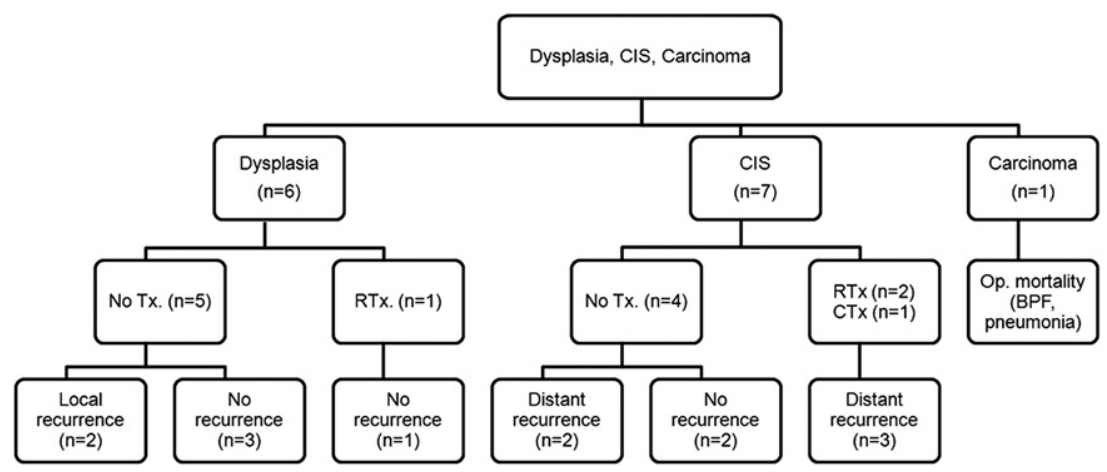

FIGURE E3. The outcomes for the patients who showed dysplasia, carcinoma in situ and carcinoma in the permanent pathology reports. $C I S$, Carcinoma in situ; $B P F$, bronchopleural fistula; $T x$, therapy; $R T x$, radiotherapy; $C T x$, chemotherapy. 

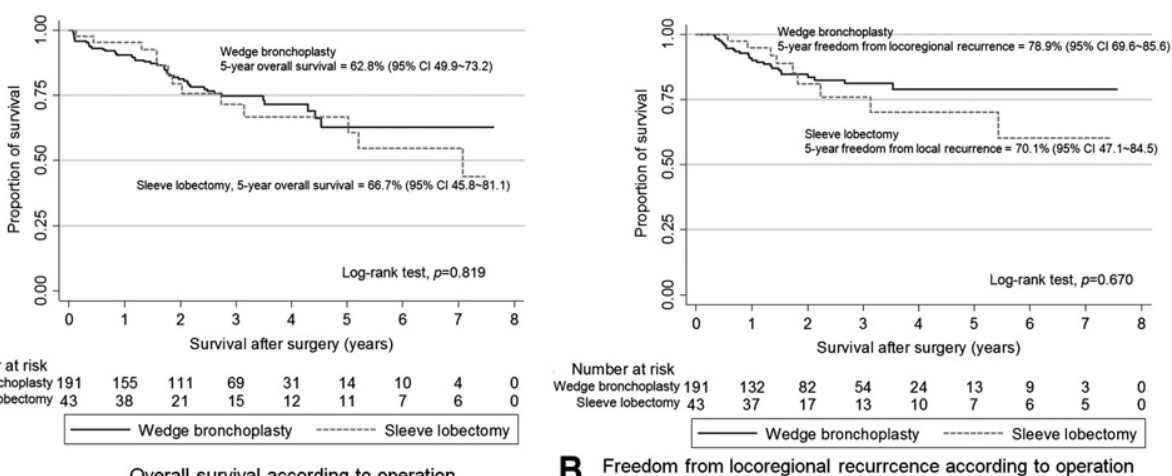

B Freedom from locoregional recurrcence according to operation

FIGURE E4. Survival curves between wedge bronchoplasty and sleeve lobectomy. A, Overall survival according to the surgical approaches. B, Freedom from locoregional recurrence according to the surgical approaches. $C I$, Confidence intervals.

TABLE E1. The agreement and disagreement between the frozen and permanent pathologic reports

\begin{tabular}{lccccc}
\hline \multirow{2}{*}{$\begin{array}{c}\text { Intraoperative frozen pathologic } \\
\text { reports }\end{array}$} & \multicolumn{4}{c}{ Permanent pathologic reports } \\
\cline { 2 - 6 } & $(-)$ for malignancy & Metaplasia & Dysplsia & CIS & Carcinoma \\
\hline$(-)$ for malignancy $(\mathrm{n}=184)$ & 175 & 2 & 2 & 4 & 1 \\
Dysplasia $(\mathrm{n}=4)$ & - & - & 3 & 1 & - \\
CIS $(\mathrm{n}=3)$ & - & - & 1 & 2 & 7 \\
& 175 & 2 & 6 & 1 \\
\hline
\end{tabular}

CIS, Carcinoma in situ. 